\title{
How common are single gene mutations as a cause for lacunar stroke?
}

\section{A targeted gene panel study}

\begin{abstract}
Rhea Y.Y. Tan, PhD, Matthew Traylor, PhD, Karyn Megy, PhD, Daniel Duarte, MSc, Sri V.V. Deevi, PhD, Olga Shamardina, PhD, and Rutendo P. Mapeta, BSc, NIHR BioResource: Rare Diseases Consortium Willem H. Ouwehand, FMedSci, Stefan Gräf, PhD, Kate Downes, PhD, and Hugh S. Markus, FMedSci
\end{abstract}

Neurology ${ }^{\circledR}$ 2019;93:e2007-e2020. doi:10.1212/WNL.0000000000008544

\section{Abstract}

\section{Objectives}

To determine the frequency of rare and pertinent disease-causing variants in small vessel disease (SVD)-associated genes (such as NOTCH3, HTRA1, COL4A1, COL4A2, FOXC1, TREX1, and GLA) in cerebral SVD, we performed targeted gene sequencing in 950 patients with younger-onset apparently sporadic SVD stroke using a targeted sequencing panel.

\section{Methods}

We designed a high-throughput sequencing panel to identify variants in 15 genes ( 7 known SVD genes, 8 SVD-related disorder genes). The panel was used to screen a population of 950 patients with younger-onset ( $\leq 70$ years) MRI-confirmed SVD stroke, recruited from stroke centers across the United Kingdom. Variants were filtered according to their frequency in control databases, predicted effect, presence in curated variant lists, and combined annotation dependent depletion scores. Whole genome sequencing and genotyping were performed on a subset of patients to provide a direct comparison of techniques. The frequency of known disease-causing and pertinent variants of uncertain significance was calculated.

\section{Results}

We identified previously reported variants in 14 patients (8 cysteine-changing NOTCH3 variants in 11 patients, 2 HTRA1 variants in 2 patients, and 1 missense COL4A1 variant in 1 patient). In addition, we identified 29 variants of uncertain significance in 32 patients.

\section{Conclusion}

Rare monogenic variants account for about $1.5 \%$ of younger onset lacunar stroke. Most are cerebral autosomal dominant arteriopathy with subcortical infarcts and leukoencephalopathy variants, but the second most common gene affected is HTRA1. A high-throughput sequencing technology platform is an efficient, reliable method to screen for such mutations.

\author{
Correspondence \\ Dr. Rhea Y.Y. Tan \\ yyrt2@medschl.cam.ac.uk
}

From the Stroke Research Group, Department of Clinical Neurosciences (R.Y.Y.T., M.T., H.S.M.), Department of Haematology (K.M., D.D., S.V.V.D., O.S., R.P.M., W.H.O., S.G., K.D.), and Division of Respiratory Medicine, Department of Medicine (S.G.), University of Cambridge; and NIHR BioResource: Rare Diseases (K.M., S.V.V.D., O.S., R.P.M., W.H.O., S.G., H.S.M.), Biomedical Campus, Cambridge, UK.

Go to Neurology.org/N for full disclosures. Funding information and disclosures deemed relevant by the authors, if any, are provided at the end of the article. 


\section{Glossary}

CADASIL = cerebral autosomal dominant arteriopathy with subcortical infarcts and leukoencephalopathy; CARASIL = cerebral autosomal recessive arteriopathy with subcortical infarcts and leukoencephalopathy; $\mathbf{C I}=$ confidence interval; $\mathbf{C N V}=$ copy number variant; DHPLC = denaturing high-performance liquid chromatography; FLAIR = fluid-attenuated inversion recovery; gnomAD = genome aggregation database; HTS = high-throughput sequencing; $\mathbf{I C H}=$ intracerebral hemorrhage; NIHR = National Institute for Health Research; SVD = small vessel disease; WES = whole-exome sequencing; WGS = wholegenome sequencing; $\mathbf{W M H}=$ white matter hyperintensities.

Cerebral small vessel disease (SVD) accounts for around $25 \%$ of strokes in the form of lacunar strokes and deep intracerebral hemorrhages (ICH) ${ }^{1}$ and is the primary pathology underlying vascular cognitive impairment. ${ }^{2}$ In the majority of cases, it is a sporadic disease of aging related to hypertension and subsequent arteriosclerosis, but a minority of cases are due to rare genetic variants. ${ }^{3}$ The most common inherited form of SVD is cerebral autosomal dominant arteriopathy with subcortical infarcts and leukoencephalopathy (CADASIL) due to NOTCH3 variants. ${ }^{4}$ More recently, other genes have been reported to cause similar phenotypes, including HTRA1, COL4A1, COL4A2, TREX1, GLA, and FOXC1. ${ }^{5}$ However, the frequency of these variants in populations with presumed sporadic SVD is unknown.

Identification of disease-causing variants currently largely relies on Sanger sequencing of the gene of interest. Often for cost reasons this involves sequencing a subset of exons, such as in CADASIL, where exons 3 and 4 are most frequently affected, and therefore preferentially screened. ${ }^{6}$ As the spectrum of monogenic SVD expands, testing on a gene-by-gene basis is not cost- or time-effective. High-throughput sequencing (HTS) panels using next-generation sequencing technologies allow simultaneous testing in multiple genes underlying a single disease phenotype in a more cost-effective manner and are increasingly being used in clinical practice.

In this study, we developed a HTS panel comprising 15 genes linked to the SVD phenotype. We evaluated the platform for disease diagnosis and to determine the frequency of monogenic disease-causing variants in a well-defined population with MRI-confirmed younger-onset lacunar stroke. This study evaluates both known disease-causing mutations and novel, potentially disease-causing variants.

\section{Methods}

\section{Platform design}

The gene panel was developed to include 7 genes known to be causal of SVD (NOTCH3, HTRA1, FOXC1, COL4A1, COL4A2, TREX1, GLA) as well as 8 genes associated with disorders with SVD-related phenotypes. These include familial cerebral amyloid angiopathy (APP, CST3, ITM2B), familial hemiplegic migraine (ATP1A2, CACNA1A, SCN1A), and connective tissue disorders (ABCC6, COL3A1). These disorders share clinical manifestations with monogenic forms of SVD (for example, lacunar stroke, MRI white matter hyperintensities $[\mathrm{WMH}]$, dementia, migraine with aura, and encephalopathy) and could therefore present similarly. For each gene, the transcript on which to report variants was selected based on size, RefSeq information, and previously reported variants, and submitted to the Locus Reference Genomic database. ${ }^{7}$ The capture design has previously been described by Simeoni et al. ${ }^{8}$

\section{Study population}

The study population consisted of patients from the UK DNA Lacunar Stroke Study. ${ }^{9}$ A total of 72 specialist centers across the United Kingdom recruited unrelated patients of European ancestry with MRI-confirmed lacunar stroke occurring at or before the age of 70 . The study was approved by the MultiCentre Research Ethics Committee for Scotland (04/ MRE00/36) and informed consent was obtained from participants. Stored DNA was available for 950 patients, all of whom were included in this study.

Lacunar stroke was defined as a clinical lacunar syndrome, with an anatomically compatible lesion on MRI (subcortical infarct $\leq 15 \mathrm{~mm}$ in diameter). All patients underwent full stroke investigations including brain MRI, carotid artery imaging, and ECG. Echocardiography was performed when appropriate. Patients were excluded if the cause of stroke was not SVD, including stenosis $>50 \%$ in the extracranial or intracranial vessels; previous carotid endarterectomy; cardioembolic source of stroke defined according to Trial of Org 10172 in Acute Stroke Treatment criteria ${ }^{10}$ as high or moderate probability; cortical infarct on MRI; subcortical infarct $>15 \mathrm{~mm}$ in diameter, as these can be caused by embolic mechanisms (striatocapsular infarcts); and any other specific cause of stroke (e.g., lupus anticoagulant, vasculitis, dissection, known monogenic cause).

All MRI scans and clinical histories were reviewed centrally by one physician (H.S.M.). The presence and extent of WMH was graded on T2-weighted or fluid-attenuated inversion recovery (FLAIR) scans using the Fazekas scale: $0=$ none, $1=$ mild, 2 = early confluent, 3 = severe confluent, as previously described. ${ }^{11}$ Lacunar infarcts were identified as a high signal lesion on acute diffusion-weighted imaging performed within 3 weeks of acute stroke, or as a cavitated hypodense lesion on $\mathrm{T} 1$ or FLAIR sequences. Cerebral microbleeds were identified 
on gradient echo sequences. Family history was collected for first-degree relatives (table 1).

\section{Sample processing}

DNA samples were processed as previously described. ${ }^{8}$

\section{Clinical bioinformatics}

Sequence reads were processed as previously described. ${ }^{8}$ The filtering step was adapted to SVD: single nucleotide variants and indels were prioritized in the following: (1) minor allele frequencies in the genome aggregation database (gnomAD) $)^{12}$ $<0.0001$; (2) predicted impact according to SnpEff ${ }^{15}$ is high, moderate, or splice region; (3) presence in HGMD Pro (2017.2) or in curated locus-specific databases (in particular the Leiden Open-Source Variation Database [May 2017

Table 1 Study population

\begin{tabular}{|c|c|}
\hline & Values \\
\hline \multicolumn{2}{|l|}{ Age at first stroke, $y$} \\
\hline Mean (SD) & $\begin{array}{l}56.3 \\
(8.7)\end{array}$ \\
\hline Median & 57.4 \\
\hline Male sex, n (\%) & $\begin{array}{l}674 \\
(70.9)\end{array}$ \\
\hline BMI, mean (SD) & $\begin{array}{l}28.7 \\
(6.2)\end{array}$ \\
\hline \multicolumn{2}{|l|}{ Number (\%) of patients with the following: } \\
\hline Hypertension & $\begin{array}{l}679 \\
(71.5)\end{array}$ \\
\hline Diabetes & $\begin{array}{l}157 \\
(16.5)\end{array}$ \\
\hline Hyperlipidemia & $\begin{array}{l}643 \\
(67.7)\end{array}$ \\
\hline Smoker (current or previous) & $\begin{array}{l}663 \\
(69.8)\end{array}$ \\
\hline Alcohol excess ( $\geq 20$ units/wk) & $\begin{array}{l}272 \\
(28.6)\end{array}$ \\
\hline Migraine & $\begin{array}{l}189 \\
(19.8)\end{array}$ \\
\hline Migraine with aura & $\begin{array}{l}106 \\
(11.1)\end{array}$ \\
\hline $\begin{array}{l}\text { Myocardial infarction/coronary artery bypass graft or } \\
\text { angioplasty }\end{array}$ & $31(3.3)$ \\
\hline Peripheral vascular disease & $26(2.7)$ \\
\hline Previous or recurrent strokes & $70(7.4)$ \\
\hline White matter hyperintensities (Fazekas grade $\geq 2$ ) & $\begin{array}{l}309 \\
(32.5)\end{array}$ \\
\hline Microbleeds present $^{a}$ & $58(18.1)$ \\
\hline
\end{tabular}

Abbreviation: $\mathrm{BMI}=$ body mass index.

${ }^{a}$ A total of 321 patients had gradient echo sequences performed to evaluate the presence of cerebral microbleeds. version $]^{13}$ ); (4) degree of deleteriousness according to Combined Annotation Dependent Depletion score ${ }^{14} \geq 15$. NOTCH3 variants that resulted in the gain or loss of a cysteine residue in the EGF-like repeats were automatically prioritized, as they are known to cause CADASIL. ${ }^{15}$ The resulting variants were assessed according to the American College of Medical Genetics and Genomics guidelines ${ }^{16}$ and retained if classified as pathogenic, likely pathogenic, or of unknown significance.

Large copy number variants (CNVs) were called using a custom pipeline based on ExomeDepth $1.1 .10^{19}$ as previously described. ${ }^{17}$

\section{Panel validation}

The panel was assessed by comparing the results with those obtained by 2 independent methods: whole-genome sequencing (WGS) and NOTCH3 and GLA sequencing.

Thirty-four samples sequenced using the panel were also sequenced using WGS, as part of the National Institute for Health Research (NIHR) BioResource-Rare Disease study. The NIHR BioResource projects were approved by Research Ethics Committees in the United Kingdom and appropriate national ethics authorities in non-United Kingdom enrollment centers. WGS was performed by Illumina (San Diego, CA) on HiSeqXTen generating 150 bp paired-end reads per lane with minimum coverage of $15 \mathrm{X}$ for at least $95 \%$ of the genome (30X on average). Reads were aligned to the GRCh37 build of the human genome reference using the Isaac Aligner, and variants were called using the Isaac VariantCaller. ${ }^{18}$ Variants in the 15 genes were analyzed following the same criteria as for the HTS panel.

Samples from all 950 patients had been previously screened for disease-causing $\mathrm{NOTCH} 3$ and GLA variants. ${ }^{9}$ Exons in 3, 4, 5, $6,11,18,19$, and 22 of NOTCH3 were screened using denaturing high-performance liquid chromatography (DHPLC), and in addition, exons 3 and 4 were screened using Sanger sequencing. ${ }^{9}$ Five patients with typical CADASIL-causing variants were identified. GLA was screened using high-resolution melt-curve analysis, covering all exons and intron/exon junctions, and one deep intronic region containing a known pathogenic variant. No Fabry-causing variants were identified. ${ }^{9}$

\section{Statistical analysis}

Comparisons of variant frequency between patients with and without a family history of stroke and with and without confluent WMH were performed using the Fisher exact test. Analyses were performed using $\mathrm{R}$ statistical software (version 3.5.1).

\section{Data availability}

Anonymized data will be made available upon reasonable request. 


\section{Results}

\section{Overall frequency of potentially disease- causing variants}

Previously reported disease-causing variants and novel rare variants are shown in table 2 .

In the 7 known SVD genes, known disease-causing variants were identified in 14 individuals (1.5\%); this represented 11 different mutations. The proportion of patients with a reported family history of stroke found to have mutations was higher ( 8 of 372 patients $[2.2 \%])$ than that in patients without a reported family history of stroke (6 of 578 patients $[1.0 \%])$, although this difference was not significant $(p=$ 0.18 ) (figure 1A).

In addition, we identified 31 novel rare variants in the 7 known SVD genes. These were identified in 35 individuals. Excluding 2 COL4A1 variants in 3 patients that were predicted to be benign in ClinVar (table 3), the overall frequency

Table 2 Known pathogenic NOTCH3 (ENST00000263388), HTRA1 (ENST00000368984), and COL4A1 (ENST00000375820) variants

\begin{tabular}{|c|c|c|c|c|c|c|c|c|}
\hline Gene & Sex & $\begin{array}{l}\text { Age, } \\
\text { y }\end{array}$ & Risk factors & Imaging features & FHx & Variant & $\begin{array}{l}\text { MAF in } \\
\text { gnomAD }\end{array}$ & References/remarks \\
\hline NOTCH3 & M & 61 & $\begin{array}{l}\text { HTN, HLD, DM, S, } \\
\text { BMI } 34\end{array}$ & $\begin{array}{l}\text { WMH FS 3, bilateral } \\
\text { ATP involvement, no } \\
\text { GE }\end{array}$ & N & $\begin{array}{l}\text { c.227G >A, } \\
\text { p.Cys76Tyr }\end{array}$ & - & $\begin{array}{l}41 \\
\text { p.Cys76 } \operatorname{Arg}^{42} \text { and p.Cys76Trp }{ }^{43,44} \text { also } \\
\text { reported } \\
\text { Not identified on DHPLC/Sanger } \\
\text { sequencing } \\
\text { Also detected on WGS }\end{array}$ \\
\hline
\end{tabular}

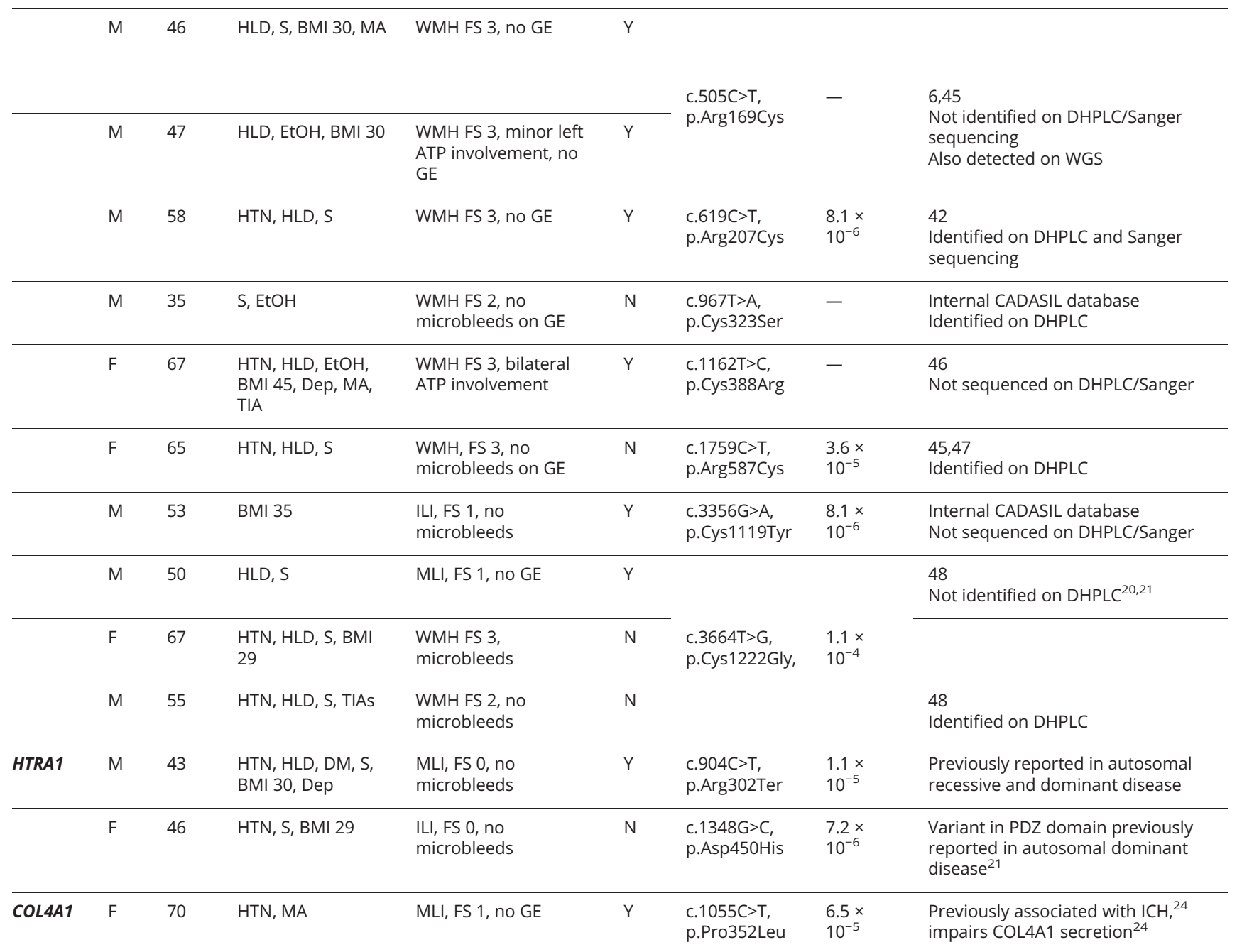

Abbreviations: ATP = anterior temporal pole; $\mathrm{BMI}=$ body mass index; CADASIL = cerebral autosomal dominant arteriopathy with subcortical infarcts and leukoencephalopathy; Dep = depression history; DHPLC = denaturing high-performance liquid chromatography; DM = diabetes mellitus; EtOH = alcohol excess; FHx = family history of stroke; FS = Fazekas score; GE = gradient echo imaging for microbleeds; gnomAD = genome aggregation database; HLD = hyperlipidemia; $\mathrm{HTN}=$ hypertension; $\mathrm{ICH}=$ intracerebral hemorrhage; ILI = isolated lacunar infarcts; $\mathrm{MA}=$ migraine with aura; $\mathrm{MAF}=$ minor allele frequencies; $\mathrm{MLI}=$ multiple lacunar infarcts; S = smoking history; WGS = whole-genome sequencing; $\mathrm{WMH}=$ white matter hyperintensities.

$\mathrm{FHx}$ was collected for first-degree relatives (parents, siblings, offspring). 


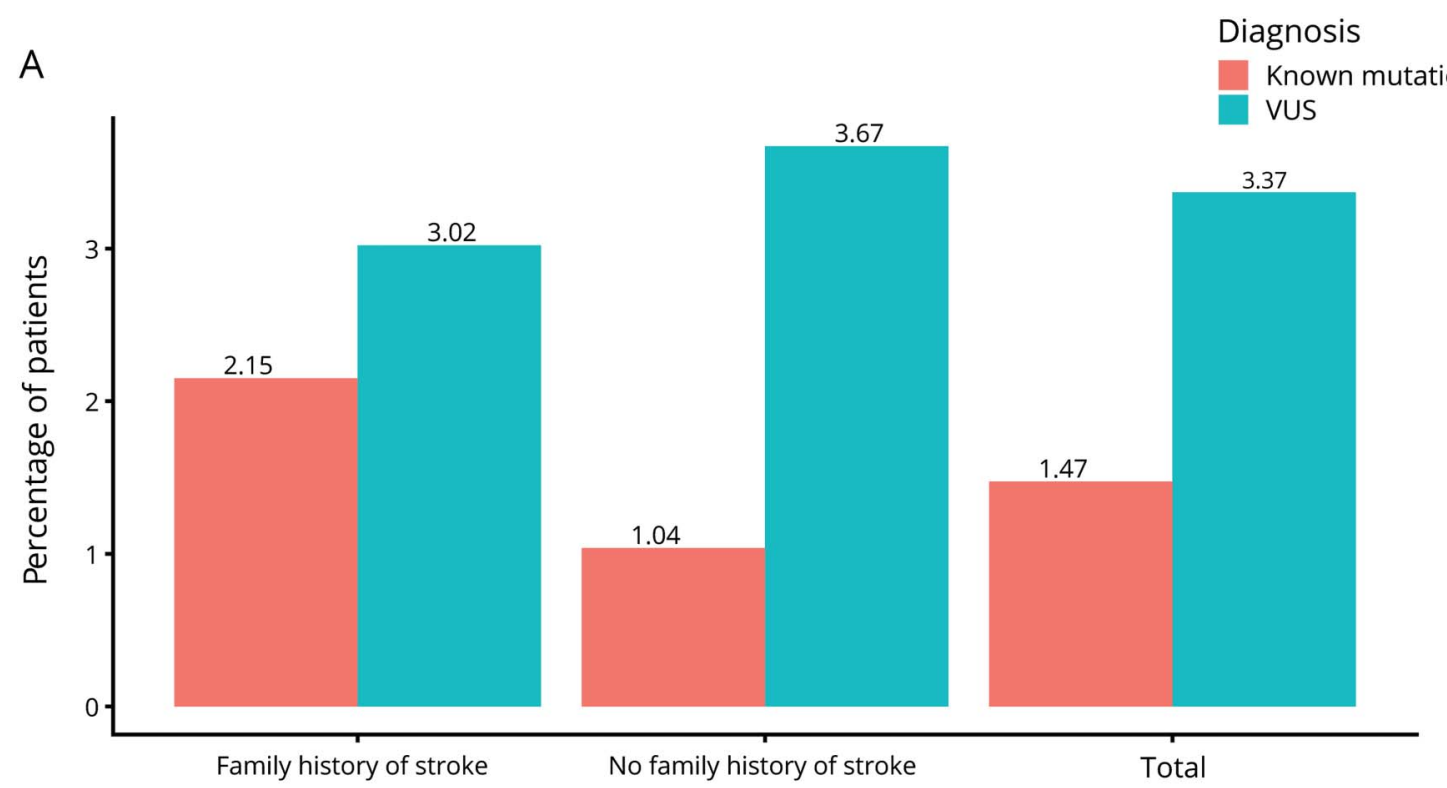

B

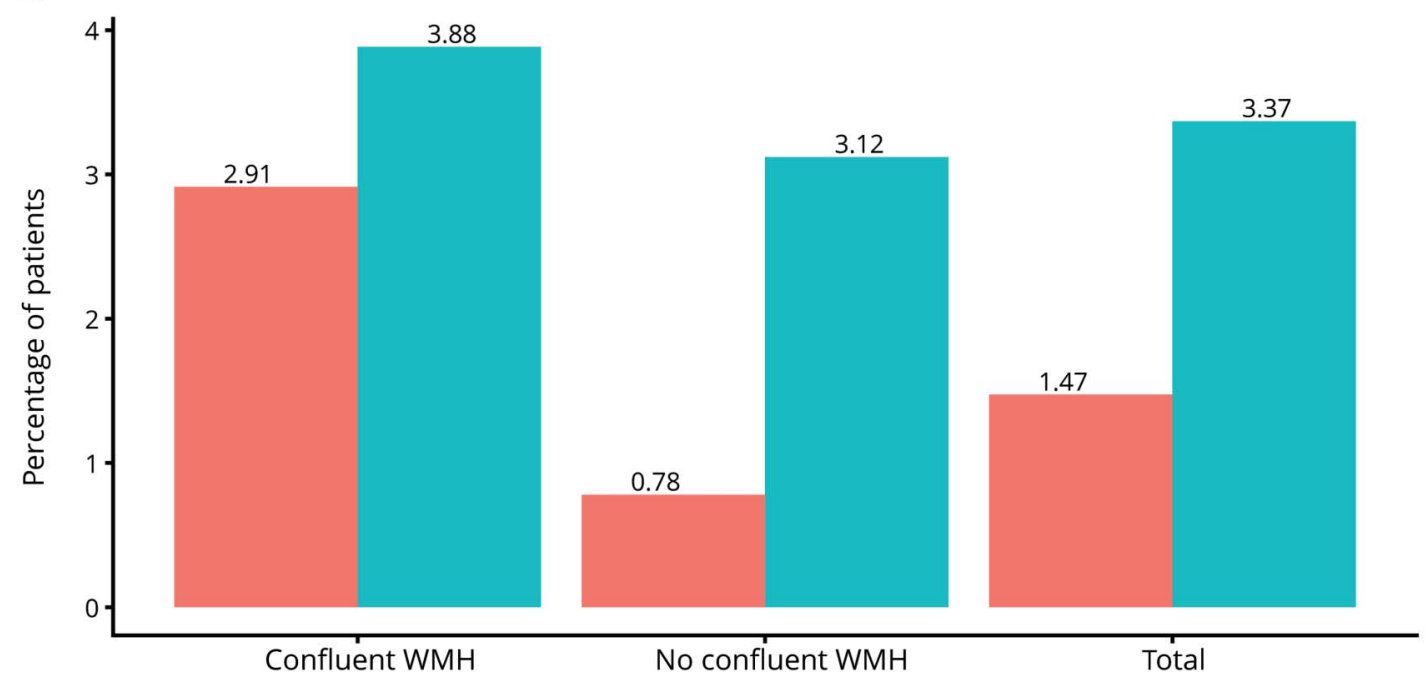

(A) Number (percentage of subjects tested) of known variants and variants of uncertain significance (VUS) identified in patients with and without a family history of stroke and (B) with and without confluent white matter hyperintensities (WMH) on MRI.

of novel variants was $3.4 \%$ ( 32 of 950 patients). There was no difference in the proportion of novel rare variants among patients with and without a family history of stroke (11 of 364 vs 21 of 572 , respectively; $p=0.71$ ) (figure $1 \mathrm{~A}$ ).

To determine whether variants were more common in a particular phenotype of SVD, or in more severe cases, we examined variant frequency in those with confluent WMH. Of the 309 patients with confluent WMH on MRI (Fazekas score ${ }^{10}$ 22), 9 (2.9\%) had a known disease-causing variant, compared with 5 of $641(0.8 \%)$ in those without confluent WMH $(p=0.018)$. The proportions for rare novel variants of uncertain significance were 12 of 309 (3.9\%) for those with WMH and 20 of $641(3.1 \%)$ for those without $(p=0.57)$ (figure 1B).

\section{CADASIL}

CADASIL is caused by cysteine-altering $\mathrm{NOTCH} 3$ mutations in the epidermal growth factor-like repeat domains encoded by exons 2 to $24 .{ }^{15}$ Eight different cysteinechanging variants in exons 2-24 of NOTCH3 were identified in 11 individuals (table 2). Previous screening had identified 5 disease-causing variants, ${ }^{9}$ and these were again identified using this platform. 
Of the 6 additional individuals with cysteine-changing NOTCH3 variants, 2 variants in 2 individuals were previously missed by both DHPLC and Sanger sequencing.

The overall frequency of CADASIL-causing variants was $1.2 \%$ (95\% confidence interval [CI] 0.6\%-2.1\%). Of patients with confluent WMH (Fazekas score $\geq 2$ ) the frequency was $2.9 \%$ (9 of $309,95 \%$ CI $1.5 \%-5.4 \%$ ) compared to $0.3 \%$ of patients without confluent WMH (Fazekas score <2) (2 of 641, 95\% CI $0.1 \%-1.1 \%)(p=0.001)$.

Comparing age groups, the overall frequency of CADASILcausing variants was $1.2 \%(95 \% \mathrm{CI} 0.6 \%-2.5 \%)$ in patients $\leq 60$ years and $1.1 \%(95 \%$ CI $0.4 \%-0.7 \%)$ in patients aged $>60$ years. Among patients with confluent WMH, the mutation frequency was $3.7 \%(95 \%$ CI $1.6 \%-8.3 \%)$ in patients $\leq 60$ years and $2.3 \%$ (95\% CI $0.9 \%-5.8 \%)$ in patients aged $>60$ years.

\section{Non-CADASIL monogenic small vessel arteriopathies}

\section{HTRA1}

Missense and nonsense HTRA1 variants have been reported in cerebral autosomal recessive arteriopathy with subcortical infarcts and leukoencephalopathy (CARASIL). ${ }^{19}$ Recently, heterozygous variants have also been identified in patients with an autosomal dominant form of SVD. ${ }^{20,21}$ Eight heterozygous missense variants and 1 nonsense HTRA 1 variant were identified in 12 individuals (1.3\%, 95\% CI 0.7\%-2.2\%). Two of these have previously been reported as disease-causing: p.Arg302Ter was reported in both recessive and dominant disease ${ }^{19,20}$; p.Asp450His was reported in autosomal dominant disease. ${ }^{21}$ p.Arg302Ter has been demonstrated to result in a mutant protein with $21 \%-50 \%$ of normal protease activity. ${ }^{19}$ There were no individuals with compound heterozygous or homozygous HTRA1 variants.

Of the remaining novel HTRA1 variants, 5 resided in the trypsin domain, with 1 (p.Arg227Trp) identified in 4 individuals. This variant is close to the trypsin active site in position 220. The clinical features of patients with HTRA1 variants are provided in table 3 .

Among patients with confluent WMH (Fazekas score $\geq 2$ ), the frequency of rare HTRA1 variants passing filters was 1.3\% (4 of $309,95 \%$ CI $0.5 \%-3.3 \%)$, similar to that in those without confluent WMH (1.2\%, 8 of $641,95 \%$ CI $0.6 \%-2.4 \%$, nonsignificant difference). In younger patients ( $\leq 60$ years), the frequency was $1.2 \%$ ( 7 of $574,95 \%$ CI $0.6 \%-2.5 \%$ ), and this value was similar in those older than 60 ( 5 of 376, 1.3\%, 95\% CI 0.5\%-3.1\%).

\section{COL4A1 and COLAA2}

COL $4 A 1$ and COL4A2 encode the $\alpha 1$ and $\alpha 2$ chains of collagen IV, respectively. Missense variants, typically but not always affecting the glycine residue in the repetitive Gly-X-Y regions, are associated with SVD and ischemic and hemorrhagic subcortical lacunar strokes. ${ }^{22}$ Variants affecting the C4 domain associated with tropocollagen assembly have also been associated with SVD. ${ }^{23}$ In 10 individuals $(1.1 \%, 95 \% \mathrm{CI}$ $0.6 \%-1.9 \%)$, we identified 9 missense COL $4 A 1$ variants (figure $2 \mathrm{~B}$ ). There were no compound heterozygous individuals. One variant (p.Pro352Leu) was previously described in an individual with presumed sporadic $\mathrm{ICH}$, and was also demonstrated to impair COL4A1 secretion into the extracellular space. ${ }^{24}$ Two other variants have been reported as likely benign in ClinVar (p.Gly332Arg and p.Pro1337Leu).

We identified 9 heterozygous missense COL4A2 variants in 9 individuals $(0.9 \%, 95 \%$ CI $0.5 \%-1.8 \%)$. None of these have previously been reported (figure 2C).

Variants identified in both COL $4 A 1$ and COL $4 A 2$ were found in various regions including the Gly-X-Y regions and the $\mathrm{C} 4$ domain associated with disease (table 3 ).

\section{FOXC1}

The FOXC1 gene encodes the Forkhead box $\mathrm{C} 1$ transcription factor. Autosomal dominant missense, nonsense, and frameshift variants, as well as deletion or duplication of the locus (6p25), have been associated with ocular abnormalities described as the Axenfeld-Rieger syndrome. ${ }^{25,26}$ Some variants are also associated with white matter abnormalities. ${ }^{25}$ Two heterozygous predicted high-impact variants in FOXC1 were identified in 2 individuals $(0.2 \%, 95 \%$ CI $0.06 \%-0.8 \%)$ : 1 novel in-frame insertion (p.Thr68_Pro69insThrProGln) and 1 frameshift variant (p.Ala381_Gly382fs) (table 3). The in-frame insertion was in a low-complexity region near the forkhead box domain, and has previously been reported in ClinVar as likely pathogenic for Axenfeld-Rieger syndrome (p.Ala381GlyfsTer147). Loss-of-function variants in FOXC1 are known to be pathogenic and the frameshift variant is predicted to lead to premature stop codon 147 residues downstream.

\section{TREX1}

Retinal vasculopathy with cerebral leukodystrophy and systemic manifestations arises due to frameshift variants near the C-terminus of TREX1. ${ }^{27}$ Missense TREX1 variants are also associated with Aicardi-Goutières syndrome, ${ }^{28}$ a form of pediatric-onset encephalopathy, and familial chilblain lupus. ${ }^{29}$ Three novel missense variants (p.Gly197Ala, p.Arg229Gly, p.Lys230Asn), 1 novel in-frame deletion, and 1 previously reported frameshift variant were found in 5 individuals $(0.5 \%$, 95\% CI 0.2\%-1.2\%). The frameshift variant (p.Ala194fs) was also identified in the same patient in a separate study using Sanger sequencing (reported as p.Ala139Valfs ${ }^{*} 21$ on a different isoform), and was found to nearly completely abolish TREX1 nuclease activity in vitro. ${ }^{30}$ This variant has been reported in a compound heterozygous case of AicardiGoutières syndrome in the DECIPHER database (patient 303873)..$^{31}$ (table 3).

\section{Fabry disease: GLA variants}

No pathogenic or likely pathogenic Fabry variants were identified. 
Table 3 Rare, novel, or presumed benign variants identified in HTRA1 (ENST00000368984), COL4A1 (ENST00000375820), COL4A2 (ENST00000360467), FOXC1 (ENST00000380874), and TREX1 (ENST00000422277) genes

\begin{tabular}{|c|c|c|c|c|c|c|c|c|}
\hline Gene & Sex & Age, y & $\begin{array}{l}\text { Risk } \\
\text { factors }\end{array}$ & Imaging features & $\begin{array}{l}\text { Family } \\
\text { history of } \\
\text { stroke }\end{array}$ & Gene/variant & $\begin{array}{l}\text { MAF in } \\
\text { gnomAD }\end{array}$ & Pathogenicity \\
\hline \multirow[t]{10}{*}{ HTRA1 } & $\mathrm{F}$ & 68 & HLD, S & $\begin{array}{l}\text { ILI, FS 1, no } \\
\text { microbleeds }\end{array}$ & $\mathrm{N}$ & $\begin{array}{l}\text { c.521A }>\text { C, } \\
\text { p.Asp174Ala }\end{array}$ & $\begin{array}{l}4.1 \times \\
10^{-6}\end{array}$ & Novel variant ${ }^{a}$ \\
\hline & $\mathrm{M}$ & 56 & $\begin{array}{l}\text { HLD, S, } \\
\text { EtOH, Dep }\end{array}$ & $\begin{array}{l}\text { MLI, FS 1, no } \\
\text { microbleeds }\end{array}$ & $\mathrm{N}$ & $\begin{array}{l}\text { c.632A>C, } \\
\text { p.Glu211Ala }\end{array}$ & - & Novel variant ${ }^{a}$ \\
\hline & $\mathrm{M}$ & 59 & $\begin{array}{l}\text { HTN, HLD, } \\
\text { DM }\end{array}$ & WMH FS 3, no GE & $\mathrm{N}$ & \multirow{4}{*}{$\begin{array}{l}\text { c. } 679 C>T, \\
\text { p.Arg227Trp }\end{array}$} & \multirow{4}{*}{$\begin{array}{l}7.9 \times \\
10^{-5}\end{array}$} & \multirow{4}{*}{ Novel variant ${ }^{b}$} \\
\hline & $\mathrm{M}$ & 62 & $\begin{array}{l}\text { HTN, HLD, } \\
\text { S, BMI 30, } \\
\text { Mig }\end{array}$ & $\begin{array}{l}\text { ILI, FS 0, no } \\
\text { microbleeds }\end{array}$ & $Y$ & & & \\
\hline & $\mathrm{M}$ & 60 & $\begin{array}{l}\text { HTN, HLD, } \\
\text { EtOH, BMI } \\
40\end{array}$ & WMH, FS 3, no GE & $\mathrm{N}$ & & & \\
\hline & $\mathrm{M}$ & 61 & $\begin{array}{l}\text { HTN, HLD, } \\
\text { S, EtOH }\end{array}$ & $\begin{array}{l}\text { ILI, FS 0, no } \\
\text { microbleeds }\end{array}$ & $\mathrm{N}$ & & & \\
\hline & $\mathrm{M}$ & 50 & HTN, S & WMH FS 3, no GE & Y & $\begin{array}{l}\text { c.834C>A, } \\
\text { p.Phe278Leu }\end{array}$ & - & Novel variant ${ }^{\mathrm{b}}$ \\
\hline & $\mathrm{M}$ & 63 & $\begin{array}{l}\text { HTN, HLD, } \\
\text { MA }\end{array}$ & $\begin{array}{l}\text { ILI, FS 1, } \\
\text { microbleeds }\end{array}$ & $\mathrm{N}$ & $\begin{array}{l}\text { c.940A }>\mathrm{G}, \\
\text { p.Met314Val }\end{array}$ & $\begin{array}{l}8.1 \times \\
10^{-6}\end{array}$ & Novel variant ${ }^{\mathrm{b}}$ \\
\hline & $\mathrm{M}$ & 41 & None & ILI, FS 0, no GE & $\mathrm{N}$ & $\begin{array}{l}\text { c.958G }>\text { A, } \\
\text { p.Asp320Asn }\end{array}$ & $\begin{array}{l}1.2 \times \\
10^{-5}\end{array}$ & Novel variant ${ }^{b}$ \\
\hline & $\mathrm{F}$ & 62 & $\begin{array}{l}\text { HLD, BMI } \\
36\end{array}$ & WMH, FS 2, no GE & Y & $\begin{array}{l}\text { c. } 1103 A>G, \\
\text { p.His368Arg }\end{array}$ & - & $\begin{array}{l}\text { Novel variant found between } \\
\text { trypsin and PDZ domains }\end{array}$ \\
\hline \multirow[t]{10}{*}{ COL4A1 } & $\mathrm{F}$ & 53 & $\begin{array}{l}\text { HTN, HLD, } \\
\text { S, EtOH, D }\end{array}$ & WMH FS 2, no GE & $Y$ & $\begin{array}{l}\text { c.994G>C, } \\
\text { p.Gly332Arg }\end{array}$ & $\begin{array}{l}4.3 \times \\
10^{-5}\end{array}$ & $\begin{array}{l}\text { ClinVar: likely benign variant; } \\
\text { ncbi.nlm.nih.gov/clinvar/ } \\
\text { variation/311067/ }\end{array}$ \\
\hline & $\mathrm{F}$ & 45 & BMI 37 & ILI, FS 0, no GE & $\mathrm{N}$ & $\begin{array}{l}\text { c. } 1246 C>G, \\
\text { p.Pro416Ala }\end{array}$ & $\begin{array}{l}4.9 \times \\
10^{-5}\end{array}$ & Novel variant ${ }^{\mathrm{d}}$ \\
\hline & $\mathrm{F}$ & 63 & $\begin{array}{l}\text { HTN, HLD, } \\
\text { S, BMI 32, } \\
\text { Dep, MA }\end{array}$ & WMH, FS 2, no GE & $\mathrm{N}$ & $\begin{array}{l}\text { c. } 2093 A>G, \\
\text { p.Lys698Arg }\end{array}$ & $\begin{array}{l}5.9 \times \\
10^{-5}\end{array}$ & Novel variant ${ }^{a}$ \\
\hline & $\mathrm{F}$ & 55 & $\begin{array}{l}\text { HTN, HLD, } \\
\text { S, BMI } 32\end{array}$ & ILI, FS 0, no GE & NK & $\begin{array}{l}\text { c. } 2174 C>T \text {, } \\
\text { p.Pro } 725 \text { Leu }\end{array}$ & - & Novel variant ${ }^{c}$ \\
\hline & $\mathrm{M}$ & 42 & $\begin{array}{l}\text { EtOH, BMI } \\
33\end{array}$ & ILI, FS 0, no GE & $\mathrm{N}$ & \multirow{3}{*}{$\begin{array}{l}\text { c.4010C>T, } \\
\text { p.Pro1337Leu }\end{array}$} & \multirow{3}{*}{$\begin{array}{l}7.9 \times \\
10^{-5}\end{array}$} & \multirow{3}{*}{$\begin{array}{l}\text { ClinVar: likely benign variant in } \\
\text { context of COL4A1-related SVD; } \\
\text { ncbi.nlm.nih.gov/clinvar/ } \\
\text { variation/311030/ }\end{array}$} \\
\hline & & & & & & & & \\
\hline & $\mathrm{F}$ & 68 & HLD & $\begin{array}{l}\text { ILI, FS 0, no } \\
\text { microbleeds }\end{array}$ & $\mathrm{N}$ & & & \\
\hline & $\mathrm{M}$ & 38 & $\begin{array}{l}\text { HTN, S, } \\
\text { BMI } 30\end{array}$ & ILI, FS 0, no GE & NK & $\begin{array}{l}\text { c. } 4423 \mathrm{~T}>\mathrm{C} \\
\text { p.Tyr1475His }\end{array}$ & $\begin{array}{l}1.6 \times \\
10^{-5}\end{array}$ & Novel variant ${ }^{\mathrm{e}}$ \\
\hline & $\mathrm{M}$ & 65 & $\mathrm{~S}$ & WMH, FS 3, no GE & $\mathrm{Y}$ & $\begin{array}{l}\text { c.4678G>A, } \\
\text { p.Val1560Met }\end{array}$ & $\begin{array}{l}1.2 \times \\
10^{-5}\end{array}$ & Novel variant ${ }^{\mathrm{e}}$ \\
\hline & $\mathrm{M}$ & 69 & $\mathrm{~S}, \mathrm{EtOH}$ & $\begin{array}{l}\text { WMH, FS 2, no } \\
\text { microbleeds }\end{array}$ & $\mathrm{N}$ & $\begin{array}{l}\text { c. } 4970 \text { C }>\text { T, } \\
\text { p.Thr1657Met }\end{array}$ & $\begin{array}{l}2.4 \times \\
10^{-5}\end{array}$ & Novel variant ${ }^{\mathrm{e}}$ \\
\hline \multirow[t]{3}{*}{ COL4A2 } & $\mathrm{M}$ & 59 & $\begin{array}{l}\text { HTN, HLD, } \\
\text { S, EtOH, } \\
\text { BMI } 37\end{array}$ & WMH, FS 2, no GE & Y & $\begin{array}{l}\text { c.661C>A, } \\
\text { p.Pro221Thr }\end{array}$ & $\begin{array}{l}1.5 \times \\
10^{-5}\end{array}$ & Novel variant ${ }^{c}$ \\
\hline & $\mathrm{M}$ & 58 & HLD & $\begin{array}{l}\text { WMH, FS 3, no } \\
\text { microbleeds }\end{array}$ & $\mathrm{N}$ & $\begin{array}{l}\text { c. } 965 G>A, \\
\text { p.Arg322GIn }\end{array}$ & $\begin{array}{l}3.6 \times \\
10^{-5}\end{array}$ & Novel variant ${ }^{c}$ \\
\hline & $\mathrm{M}$ & 65 & $\begin{array}{l}\text { HTN, HLD, } \\
\text { S }\end{array}$ & ILI, FS 0, no GE & NK & $\begin{array}{l}\text { c.1396G>A, } \\
\text { p.Gly466Ser }\end{array}$ & $\begin{array}{l}5.4 \times \\
10^{-5}\end{array}$ & Novel variant ${ }^{c}$ \\
\hline
\end{tabular}


Table 3 Rare, novel, or presumed benign variants identified in HTRA1 (ENST00000368984), COL4A1 (ENST00000375820), COL4A2 (ENST00000360467), FOXC1 (ENST00000380874), and TREX1 (ENST00000422277) genes (continued)

\begin{tabular}{|c|c|c|c|c|c|c|c|c|}
\hline Gene & Sex & Age, y & $\begin{array}{l}\text { Risk } \\
\text { factors }\end{array}$ & Imaging features & $\begin{array}{l}\text { Family } \\
\text { history of } \\
\text { stroke }\end{array}$ & Gene/variant & $\begin{array}{l}\text { MAF in } \\
\text { gnomAD }\end{array}$ & Pathogenicity \\
\hline & M & 56 & $\begin{array}{l}\text { HTN, HLD, } \\
\text { BMI } 29\end{array}$ & ILI, FS 1, no GE & NK & $\begin{array}{l}\text { c.1687G>A, } \\
\text { p.Gly563Ser }\end{array}$ & - & Novel variant ${ }^{\mathrm{d}}$ \\
\hline & $\mathrm{M}$ & 53 & $\begin{array}{l}\text { HLD, S, BMI } \\
40\end{array}$ & ILI, FS 0, no GE & Y & $\begin{array}{l}\text { c. } 2581 C>T, \\
\text { p.Arg861Cys }\end{array}$ & $\begin{array}{l}2.7 \times \\
10^{-5}\end{array}$ & Novel variant ${ }^{\mathrm{c}}$ \\
\hline & $\mathrm{M}$ & 65 & $\mathrm{EtOH}$ & WMH, FS 2, no GE & NK & $\begin{array}{l}\text { c.3715G>A, } \\
\text { p.Gly1239Ser }\end{array}$ & - & Novel variant ${ }^{\mathrm{d}}$ \\
\hline & $\mathrm{M}$ & 61 & $\begin{array}{l}\text { HTN, HLD, } \\
\text { S, BMI } 30\end{array}$ & ILI, FS 1, no GE & $\mathrm{Y}$ & $\begin{array}{l}\text { c.4292G >A, } \\
\text { p.Arg1431His }\end{array}$ & $\begin{array}{l}2.9 \times \\
10^{-5}\end{array}$ & Novel variant ${ }^{\mathrm{d}}$ \\
\hline & $\mathrm{M}$ & 62 & HTN, S & MLI, FS 0, no GE & $\mathrm{N}$ & $\begin{array}{l}\text { c. } 4534 \mathrm{~T}>\mathrm{C}, \\
\text { p.Trp1512Arg }\end{array}$ & $\begin{array}{l}6.2 \times \\
10^{-5}\end{array}$ & Novel variant ${ }^{\mathrm{e}}$ \\
\hline & $\mathrm{M}$ & 58 & $\begin{array}{l}\text { HTN, HLD, } \\
\text { DM, BMI } \\
\text { 34, Dep }\end{array}$ & MLI, FS 0, no GE & Y & $\begin{array}{l}\text { c.4601C>T, } \\
\text { p.Ala1534Val }\end{array}$ & $\begin{array}{l}1.2 \times \\
10^{-5}\end{array}$ & Novel variant ${ }^{\mathrm{e}}$ \\
\hline \multirow[t]{2}{*}{ FOXC1 } & $\mathrm{M}$ & 58 & $\begin{array}{l}\text { HLD, S, BMI } \\
32\end{array}$ & $\begin{array}{l}\text { ILI, FS 0, no } \\
\text { microbleeds }\end{array}$ & $\mathrm{Y}$ & $\begin{array}{l}\text { c.204_- } \\
\text { 205insGCCGCA, } \\
\text { p.Thr68_- } \\
\text { Pro69insThr, } \\
\text { ProGIn }\end{array}$ & - & Novel in-frame insertion \\
\hline & $\mathrm{M}$ & 47 & HLD, S & ILI, FS 0, no GE & Y & $\begin{array}{l}\text { c.1141_1142insG, } \\
\text { p.Ala381_- } \\
\text { Gly382fs }\end{array}$ & - & $\begin{array}{l}\text { ClinVar: likely pathogenic variant for } \\
\text { Axenfeld-Rieger syndrome; ncbi. } \\
\text { nlm.nih.gov/clinvar/variation/ } \\
\text { 537387/; frameshift variants } \\
\text { associated with SVD. }{ }^{25}\end{array}$ \\
\hline \multirow[t]{5}{*}{ TREX1 } & $\mathrm{F}$ & 48 & $\begin{array}{l}\text { HTN, HLD, } \\
\text { DM, BMI } \\
\text { 31, Dep }\end{array}$ & $\begin{array}{l}\text { WMH FS 3, multiple } \\
\text { microbleeds }\end{array}$ & $\mathrm{N}$ & $\begin{array}{l}\text { c.581delC, } \\
\text { p.Ala194fs }\end{array}$ & $\begin{array}{l}2.8 \times \\
10^{-5}\end{array}$ & $\begin{array}{l}\text { Previously reported in Aicardi- } \\
\text { Goutières syndrome }{ }^{31} ; \text { also } \\
\text { identified in the same patient on } \\
\text { Sanger sequencing and found to } \\
\text { impair TREX1 function }\end{array}$ \\
\hline & $\mathrm{F}$ & 43 & $\begin{array}{l}\text { HTN, S, } \\
\text { BMI 30, } \\
\text { Dep }\end{array}$ & ILI, FS 0, no GE & Y & $\begin{array}{l}\text { c.590G>C, } \\
\text { p.Gly197Ala }\end{array}$ & - & Novel variant \\
\hline & $\mathrm{F}$ & 51 & $\begin{array}{l}\text { HTN, HLD, } \\
\text { DM, BMI } \\
\text { 39, Dep }\end{array}$ & WMH, FS 2, no GE & $\mathrm{N}$ & $\begin{array}{l}\text { c. } 685 A>G \text {, } \\
\text { p.Arg229Gly }\end{array}$ & $\begin{array}{l}1.1 \times \\
10^{-5}\end{array}$ & Novel variant \\
\hline & $\mathrm{M}$ & 65 & $\begin{array}{l}\text { HTN, BMI } \\
28\end{array}$ & $\begin{array}{l}\text { ILI, FS 0, no } \\
\text { microbleeds }\end{array}$ & $\mathrm{N}$ & $\begin{array}{l}\text { c.690G >T, } \\
\text { p.Lys230Asn }\end{array}$ & - & Novel variant \\
\hline & $\mathrm{F}$ & 60 & $\begin{array}{l}\text { HTN, HLD, } \\
\text { BMI } 32\end{array}$ & ILI, FS 0, no GE & $\mathrm{N}$ & $\begin{array}{l}\text { c.1024_1041del, } \\
\text { CTGCTGGCCCCA, } \\
\text { CTGGGT } \\
\text { p.Leu342_- } \\
\text { Gly347del }\end{array}$ & $\begin{array}{l}6.1 \times \\
10^{-5}\end{array}$ & Novel in-frame deletion \\
\hline
\end{tabular}

Abbreviations: $\mathrm{BMI}=$ body mass index; Dep = depression history; DM = diabetes mellitus; EtOH = alcohol excess; FS = Fazekas score; GE = gradient echo imaging for microbleeds; gnomAD = genome aggregation database; HLD = hyperlipidemia; HTN = hypertension; ILI = isolated lacunar infarcts; MA = migraine with aura; MAF = minor allele frequencies; Mig = migraine without aura; MLI = multiple lacunar infarcts; NK = not known; S = smoking history; SVD = small vessel disease; $\mathrm{WMH}=$ white matter hyperintensities.

Family history was collected for first-degree relatives (parents, siblings, offspring).

a Found between trypsin and kazal domains of HTRA1 gene, of uncertain significance.

${ }^{b}$ Found in trypsin domain of HTRA1 gene, associated with enzyme function.

' In triple helix repeat region of COL4A1 or COL4A2 genes-other Gly-altering variants associated with disease.

d In low complexity region of COL4A1 or COL4A2 genes, of uncertain significance.

e In C4 domain of COL4A1 or COL4A2 genes, associated with tropocollagen assembly, ${ }^{49}$ variants previously associated with disease. ${ }^{23}$

\section{Copy number variants}

Excluding CNVs with a Bayes factor lower than 20, only 1 CNV was detected in the 7 known cerebral SVD genes. This was a large duplication of both COL4A1 and COL4A2 in a single individual.

\section{SVD-related genes}

Forty-five heterozygous variants in 8 genes associated with SVD-related disorders were identified in 47 individuals (table 4). Of note, 2 novel variants were identified in cystatin 3 (CST3), where variants are known to cause familial 


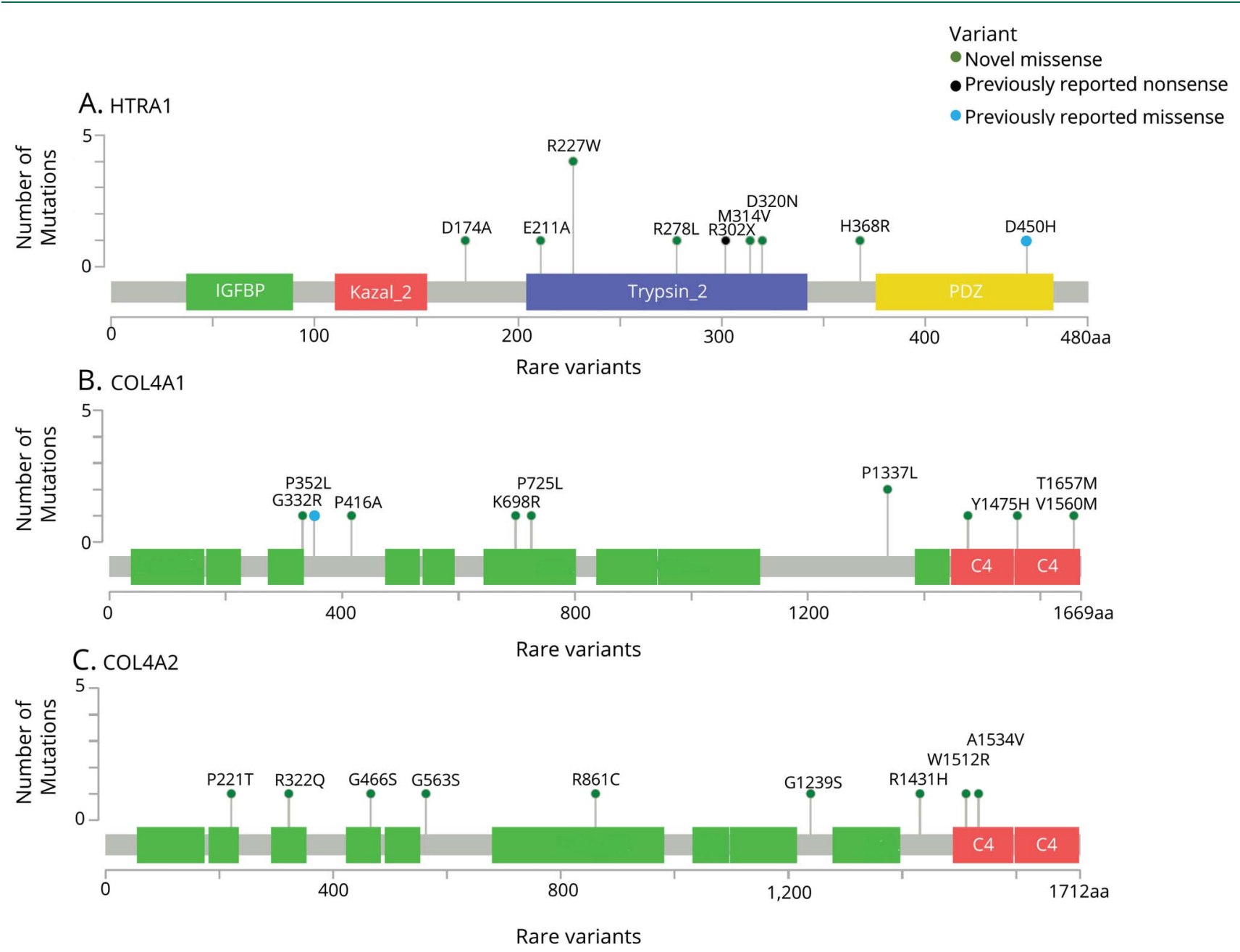

Rare variants identified in (A) HTRA1 (ENST00000368984), (B) COL4A1 (ENST00000375820), and (C) COL4A2 (ENST00000360467).

cerebral amyloid angiopathy, Icelandic type ${ }^{32}: 1$ stopgained and 1 frameshift variant, both at position 93 (p.Glu93* and p.Glu93fs). Lacunar stroke is not an established manifestation of the disease, indicating that more investigations are needed to assign pathogenicity to these variants.

\section{Validation of panel}

In the 34 individuals sequenced by WGS, 2 were found to harbor CADASIL-causing NOTCH3 variants. These were also detected by the HTS platform (table 2). No other exonic variants passing the filters could be identified in the remaining 32 individuals, either by WGS or by targeted sequencing. In addition, 5 cysteine-changing NOTCH3 variants had been detected on prior screening, all of which were also identified by the platform.

\section{Discussion}

We developed an HTS panel for 7 genes known to be implicated in monogenic SVD and 8 genes associated with related phenotypes that can enter in the differential diagnosis of monogenic SVD. Validation against other techniques found the panel to be as sensitive, if not more sensitive than conventional screening methods, and detected all previously identified NOTCH3 variants, including some missed on previous screening.

NOTCH3 variants, which underlie CADASIL, the most common cause of monogenic SVD, were identified in about $1 \%$ of patients with apparently sporadic SVD aged 70 or under. The yield rose to $3.7 \%$ if analysis was limited to younger (age $\leq 60$ ) patients who also had confluent WMH. 
Table 4 Rare variants identified in genes associated with phenotypes related to small vessel disease: COL3A1

(ENST00000304636), APP (ENST00000346798), CST3 (ENST00000376925), ITM2B (ENST00000378565), ATP1A2

(ENST00000361216), and CACNA1A (ENST00000361216), SCN1A (ENST00000375405)

\begin{tabular}{|c|c|c|c|c|c|c|c|}
\hline $\begin{array}{l}\text { Related } \\
\text { phenotype }\end{array}$ & Gene & Variant & dbSNP ID & $\begin{array}{l}\text { No. of } \\
\text { individuals }\end{array}$ & $\begin{array}{l}\text { MAF in } \\
\text { gnomAD }\end{array}$ & Remarks & Reference \\
\hline \multirow{5}{*}{$\begin{array}{l}\text { Ehlers- } \\
\text { Danlos (IV) }\end{array}$} & COL3A1 & c.74A>G, p.G $\ln 25 A r g$ & - & 1 & - & In signal peptide & \\
\hline & & $\begin{array}{l}\text { c.1856C }>\mathrm{T}, \\
\text { p.Pro619Leu }\end{array}$ & rs373838193 & 1 & $\begin{array}{l}4.3 \times \\
10^{-5}\end{array}$ & $\begin{array}{l}\text { In low complexity region } \\
\text { VUS on ClinVar for aortic aneurysm/ } \\
\text { dissection and Ehlers-Danlos } \\
\text { type IV }\end{array}$ & $\begin{array}{l}\text { ncbi.nlm.nih. } \\
\text { gov/clinvar/ } \\
\text { variation/ } \\
\text { 199718/ }\end{array}$ \\
\hline & & $\begin{array}{l}\text { c.1864C>T, } \\
\text { p.Pro622Ser }\end{array}$ & rs772638774 & 2 & $\begin{array}{l}3.3 \times \\
10^{-5}\end{array}$ & In low complexity region & \\
\hline & & c.226A>G, p.Asn76Asp & rs142045411 & 1 & $\begin{array}{l}6.2 \times \\
10^{-5}\end{array}$ & $\begin{array}{l}\text { In von Willebrand factor type C } \\
\text { domain }\end{array}$ & \\
\hline & & $\begin{array}{l}\text { c. } 1165 \mathrm{~A}>\mathrm{T} \\
\text { p.Asn389Tyr }\end{array}$ & rs200394946 & 1 & $\begin{array}{l}9.7 \times \\
10^{-5}\end{array}$ & $\begin{array}{l}\text { In collagen triple helix repeat } \\
\text { domain } \\
\text { VUS on ClinVar for aortic aneurysm/ } \\
\text { dissection and Ehlers-Danlos Type } \\
\text { IV }\end{array}$ & $\begin{array}{l}\text { ncbi.nlm.nih. } \\
\text { gov/clinvar/ } \\
\text { variation/ } \\
\text { 199718/ }\end{array}$ \\
\hline \multirow{10}{*}{$\begin{array}{l}\text { Cerebral } \\
\text { amyloid } \\
\text { angiopathy }\end{array}$} & $A P P$ & $\begin{array}{l}\text { c.373G }>\text { A, } \\
\text { p.Asp125Asn }\end{array}$ & - & 1 & - & $\begin{array}{l}\text { In amyloid A4 N-terminal heparin } \\
\text { binding domain }\end{array}$ & \\
\hline & & c.682G>A, p.Val228Ile & rs755841034 & 2 & $\begin{array}{l}4.1 \times \\
10^{-6}\end{array}$ & $\begin{array}{l}\text { In low complexity region, near } \\
\text { p.Val225Ala, a VUS associated with } \\
\text { familial Alzheimer disease }\end{array}$ & \\
\hline & & $\begin{array}{l}\text { c.727G }>\text { A, } \\
\text { p.Asp243Asn }\end{array}$ & rs750279232 & 1 & $\begin{array}{l}3.3 \times \\
10^{-6}\end{array}$ & In coiled coil domain & \\
\hline & & c.736G>A, p.Glu246Lys & rs147485129 & 1 & $\begin{array}{l}1.8 \times \\
10^{-5}\end{array}$ & In coiled coil domain & \\
\hline & & $\begin{array}{l}\text { c. } 1859 C>T, \\
\text { p.Pro620Leu }\end{array}$ & rs759926843 & 1 & $\begin{array}{l}1.6 \times \\
10^{-5}\end{array}$ & In low complexity region & \\
\hline & & $\begin{array}{l}\text { c. } 1909+5 G>A \text {, Intron } \\
\text { and splice region }\end{array}$ & rs200271509 & 1 & $\begin{array}{l}1.5 \times \\
10^{-5}\end{array}$ & In coiled coil domain & \\
\hline & & c.2148C>T, p.lle716lle & rs145564988 & 1 & $\begin{array}{l}6.9 \times \\
10^{-5}\end{array}$ & $\begin{array}{l}\text { In C-terminus domain; Ile716Thr } \\
\text { reported on ClinVar (no clinical } \\
\text { interpretation available); lle716Val } \\
\text { associated with familial Alzheimer } \\
\text { disease }\end{array}$ & $\begin{array}{l}\text { ncbi.nlm.nih. } \\
\text { gov/clinvar/ } \\
\text { variation/ } \\
98240 / \\
50\end{array}$ \\
\hline & CST3 & c.277G>T, p.Glu93Ter & - & 1 & $\begin{array}{l}4.1 \times \\
10^{-6}\end{array}$ & $\begin{array}{l}\text { p.Leu94GIn reported in familial } \\
\text { Icelandic dementia }\end{array}$ & 51 \\
\hline & & $\begin{array}{l}\text { c.277_296delGAGCT. } \\
\text { GGGCCGAACCACGTG. } \\
\text { p.Glu93fs }\end{array}$ & rs765138253 & 1 & $\begin{array}{l}6.1 \times \\
10^{-5}\end{array}$ & & \\
\hline & ITM2B & c. $92 \mathrm{C}>\mathrm{G}$, p.Pro31Arg & rs150336652 & 1 & $\begin{array}{l}8.9 \times \\
10^{-5}\end{array}$ & Low complexity region & \\
\hline \multirow[t]{3}{*}{ FHM } & ATP1A2 & $\begin{array}{l}\text { c.1262G>A, } \\
\text { p.Arg421Gln }\end{array}$ & rs139499540 & 1 & $\begin{array}{l}5.5 \times \\
10^{-5}\end{array}$ & $\begin{array}{l}\text { Between E1-E2 and Cation ATPase } \\
\text { domains } \\
\text { Reported as VUS for FHM on ClinVar }\end{array}$ & $\begin{array}{l}\text { ncbi.nlm.nih. } \\
\text { gov/clinvar/ } \\
\text { variation/ } \\
\text { 406194/ }\end{array}$ \\
\hline & & $\begin{array}{l}\text { c.2146G>T, } \\
\text { p.Val716Leu }\end{array}$ & - & 1 & - & $\begin{array}{l}\text { Near metal ion binding sites at } \\
\text { residues } 714 \text { and } 718 \\
\text { p.Asp718Asn reported as } \\
\text { pathogenic in FHM }\end{array}$ & 52 \\
\hline & CACNA1A & c.130G>A, p.Ala44Thr & rs201398669 & 1 & $\begin{array}{l}2.5 \times \\
10^{-5}\end{array}$ & Low complexity region & \\
\hline
\end{tabular}


Table 4 Rare variants identified in genes associated with phenotypes related to small vessel disease: COL3A1

(ENST00000304636), APP (ENST00000346798), CST3 (ENST00000376925), ITM2B (ENST00000378565), ATP1A2

(ENST00000361216), and CACNA1A (ENST00000361216), SCN1A (ENST00000375405) (continued)

\begin{tabular}{|c|c|c|c|c|c|c|c|}
\hline $\begin{array}{l}\text { Related } \\
\text { phenotype }\end{array}$ & Gene & Variant & dbSNP ID & $\begin{array}{l}\text { No. of } \\
\text { individuals }\end{array}$ & $\begin{array}{l}\text { MAF in } \\
\text { gnomAD }\end{array}$ & Remarks & Reference \\
\hline & & c.633T>C, p.Ser211Ser & rs202216404 & 1 & $\begin{array}{l}6.2 \times \\
10^{-5}\end{array}$ & $\begin{array}{l}\text { Ion transport domain p.Ser211Asn } \\
\text { reported as VUS for unspecified } \\
\text { phenotype }\end{array}$ & $\begin{array}{l}\text { ncbi.nlm.nih. } \\
\text { gov/clinvar/ } \\
\text { variation/ } \\
\text { 426949/ }\end{array}$ \\
\hline & & $\begin{array}{l}\text { c.1083-108_1083- } \\
\text { 101delCACACACA }\end{array}$ & - & 1 & - & Intron variant & \\
\hline & & $\begin{array}{l}\text { c.2524G >C, } \\
\text { p.Glu842Gln }\end{array}$ & - & 1 & - & $\begin{array}{l}\text { Between ion transport domains; } \\
\text { p.Ala841Ser reported as VUS for } \\
\text { unspecified phenotype }\end{array}$ & $\begin{array}{l}\text { ncbi.nlm.nih. } \\
\text { gov/clinvar/ } \\
\text { variation/ } \\
\text { 385371/ }\end{array}$ \\
\hline & & $\begin{array}{l}\text { c.2812G>C, } \\
\text { p.Gly938Arg }\end{array}$ & rs771423362 & 1 & $\begin{array}{l}4.1 \times \\
10^{-5}\end{array}$ & $\begin{array}{l}\text { Between ion transport domains; } \\
\text { reported as VUS for unspecified } \\
\text { phenotype }\end{array}$ & $\begin{array}{l}\text { ncbi.nlm.nih. } \\
\text { gov/clinvar/ } \\
\text { variation/ } \\
\text { 422044/ }\end{array}$ \\
\hline & & $\begin{array}{l}\text { c. } 2944 G>A, \\
\text { p.Gly982Ser }\end{array}$ & - & 1 & - & Low complexity region & \\
\hline & & $\begin{array}{l}\text { c.2960G>A, } \\
\text { p.Arg987Gln }\end{array}$ & - & 1 & - & $\begin{array}{l}\text { Low complexity region; } \\
\text { p.Arg987Pro reported as VUS for } \\
\text { unspecified phenotype }\end{array}$ & $\begin{array}{l}\text { ncbi.nlm.nih. } \\
\text { gov/clinvar/ } \\
\text { variation/ } \\
\text { 387025/ }\end{array}$ \\
\hline & & $\begin{array}{l}\text { c.3226G>A, } \\
\text { p.Ala1076Thr }\end{array}$ & rs554091859 & 1 & $\begin{array}{l}8.6 \times \\
10^{-5}\end{array}$ & $\begin{array}{l}\text { Between ion transport domains; } \\
\text { reported as VUS (unspecified } \\
\text { phenotype) }\end{array}$ & $\begin{array}{l}\text { ncbi.nlm.nih. } \\
\text { gov/clinvar/ } \\
\text { variation/ } \\
\text { 429962/ }\end{array}$ \\
\hline & & $\begin{array}{l}\text { c.3265G>A, } \\
\text { p.Gly1089Ser }\end{array}$ & rs201311000 & 1 & $\begin{array}{l}3.3 \times \\
10^{-5}\end{array}$ & $\begin{array}{l}\text { Between ion transport domains; } \\
\text { Gly1089Cys reported as VUS for } \\
\text { unspecified phenotype }\end{array}$ & $\begin{array}{l}\text { ncbi.nlm.nih. } \\
\text { gov/clinvar/ } \\
\text { variation/ } \\
\text { 391877/ }\end{array}$ \\
\hline & & $\begin{array}{l}\text { c.3436G }>A, \\
\text { p.Val1146lle }\end{array}$ & rs376365775 & 1 & $\begin{array}{l}1.6 \times \\
10^{-5}\end{array}$ & $\begin{array}{l}\text { Between ion transport domains; } \\
\text { reported as VUS for unspecified } \\
\text { phenotype }\end{array}$ & $\begin{array}{l}\text { ncbi.nlm.nih. } \\
\text { gov/clinvar/ } \\
\text { variation/ } \\
\text { 195470/ }\end{array}$ \\
\hline & & $\begin{array}{l}\text { c. } 4646 A>G, \\
\text { p.Gln1549Arg }\end{array}$ & rs759263620 & 1 & $\begin{array}{l}4.1 \times \\
10^{-5}\end{array}$ & Between ion transport domains & \\
\hline & & $\begin{array}{l}\text { c.5494G>, } \\
\text { p.Val1832Met }\end{array}$ & rs376815942 & 1 & $\begin{array}{l}1.2 \times \\
10^{-5}\end{array}$ & $\begin{array}{l}\text { GPHH (voltage-dependent L type } \\
\text { calcium channel) domain }\end{array}$ & \\
\hline & & $\begin{array}{l}\text { c.5795A }>\text { G, } \\
\text { p.Asn1932Ser }\end{array}$ & - & 1 & $\begin{array}{l}4.1 \times \\
10^{-6}\end{array}$ & $\begin{array}{l}\text { Voltage-gated calcium channel IQ } \\
\text { domain }\end{array}$ & \\
\hline & & $\begin{array}{l}\text { c.5936A>C, } \\
\text { p.Glu1979Ala }\end{array}$ & rs774657158 & 1 & $\begin{array}{l}8.3 \times \\
10^{-6}\end{array}$ & Outside of Ca channel IQ domain & \\
\hline & & $\begin{array}{l}\text { c. } 6400 C>T, \\
\text { p.Arg2134Cys }\end{array}$ & rs121908235 & 1 & $\begin{array}{l}6.2 \times \\
10^{-5}\end{array}$ & $\begin{array}{l}\text { Outside of Ca channel IQ domain } \\
\text { VUS for unspecified phenotype and } \\
\text { associated with episodic ataxia type } \\
2 \text { (impact uncertain) }\end{array}$ & $\begin{array}{l}\text { ncbi.nlm.nih. } \\
\text { gov/clinvar/ } \\
\text { variation/ } \\
68440\end{array}$ \\
\hline & & $\begin{array}{l}\text { c. } 6644 A>C, \\
\text { p.His2215Pro }\end{array}$ & - & 1 & $\begin{array}{l}3.8 \times \\
10^{-5}\end{array}$ & Low complexity region & \\
\hline & & $\begin{array}{l}\text { c.6715C }>\mathrm{T} \\
\text { p.Arg2239Trp }\end{array}$ & rs759576380 & 1 & $\begin{array}{l}5.2 \times \\
10^{-5}\end{array}$ & Outside of Ca channel IQ domain & \\
\hline & & $\begin{array}{l}\text { c.6758G>A, } \\
\text { p.Arg2253GIn }\end{array}$ & rs752950486 & 1 & $\begin{array}{l}1.6 \times \\
10^{-5}\end{array}$ & Outside of Ca channel IQ domain & \\
\hline & & $\begin{array}{l}\text { c.6839G }>\mathrm{T} \\
\text { p.Arg2280Leu }\end{array}$ & - & 1 & - & $\begin{array}{l}\text { Patient also has a deletion in ABCC6 } \\
\text { Outside of Ca channel IQ domain }\end{array}$ & \\
\hline & & $\begin{array}{l}\text { c.7027G >A, } \\
\text { p.Gly2343Ser }\end{array}$ & - & 1 & $\begin{array}{l}6.5 \times \\
10^{-5}\end{array}$ & Outside of Ca channel IQ domain & \\
\hline
\end{tabular}


Table 4 Rare variants identified in genes associated with phenotypes related to small vessel disease: COL3A1 (ENST00000304636), APP (ENST00000346798), CST3 (ENST00000376925), ITM2B (ENST00000378565), ATP1A2 (ENST00000361216), and CACNA1A (ENST00000361216), SCN1A (ENST00000375405) (continued)

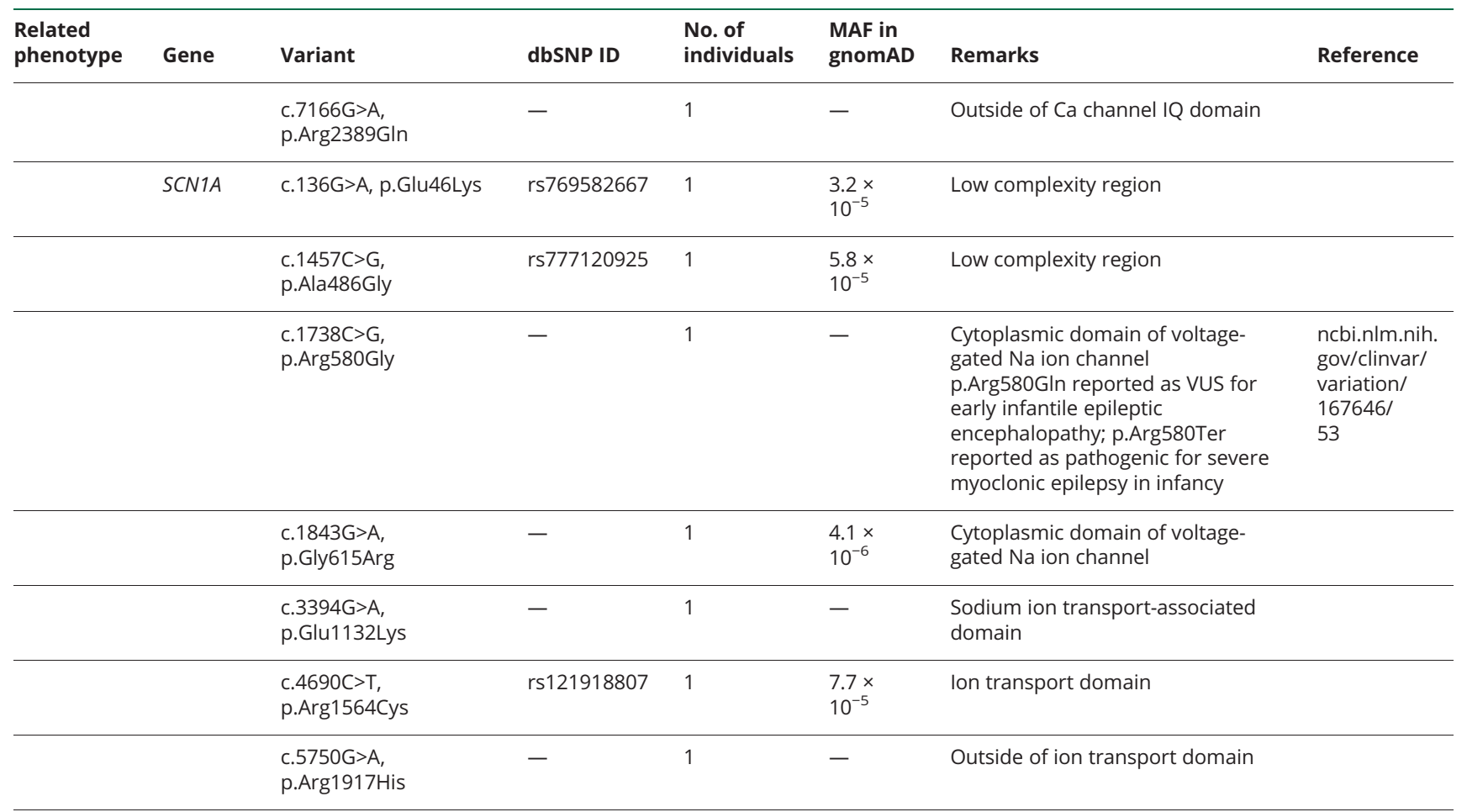

Abbreviations: $\mathrm{FHM}=$ familial hemiplegic migraine; gnomAD = genome aggregation database; $\mathrm{MAF}=$ minor allele frequency; VUS = variants of unknown significance.

A number of other monogenic forms of SVD have been reported, and although they appear to be rarer than CADASIL, their frequency in cases of lacunar stroke is unknown. Our study provides data on their prevalence in cases of symptomatic lacunar stroke.

We found no homozygous CARASIL-causing variants, but up to $1.3 \%$ of cases had potentially disease-causing heterozygous missense and nonsense variants, suggesting that HTRA1-associated autosomal dominant SVD is the second most common cause of familial SVD. These findings are consistent with previous smaller studies in which about $5 \%$ of patients with NOTCH3-negative familial SVD carry missense and nonsense HTRA1 variants. ${ }^{21,33,34}$

In addition, we identified several rare variants predicted to be damaging in COL4A1, COL4A2, and FOXC1. One individual had a $C O L 4 A 1$ variant (c.1055C $>$ T, p.Pro352Leu), which has been described in an individual with sporadic $\mathrm{ICH} .{ }^{24}$ However, without functional support and evidence of segregation with disease, the pathogenicity of this variant remains uncertain.

We identified a frameshift FOXC1 variant (c.1141 1142insG, p.Ala381_Gly382fs). Given that FOXC1 has 1 exon, the transcript containing this predicted premature stop codon may escape nonsense-mediated decay. Functional studies are required to confirm pathogenicity of this highimpact variant.

Fabry disease has been suggested as an underreported cause of young stroke, ${ }^{35}$ and has been associated with MRI features of SVD, ${ }^{36}$ but we found no Fabry variants in this cohort. This is consistent with recent data suggesting that its importance as a cause of early-onset cryptogenic stroke may have been overestimated. $^{37,38}$

Our results highlight a major challenge in the use of HTS panels in clinical practice; namely, determining whether a variant is pathogenic or benign. In CADASIL, variant interpretation is aided by the knowledge that all known diseasecausing variants are cysteine-altering. Assigning pathogenicity of variants in genes such as HTRA1, however, is challenging. Variant interpretation guidelines highlight the use of allele frequency in large public databases. This presents particular challenges in late-onset diseases such as SVD. Databases such as gnomAD, ${ }^{39}$ which are used to infer population-level frequencies of potentially disease-causing variants, include individuals drawn from different populations predominantly of European ancestry, with variable age distributions. For individuals who are young at the point of recruitment, it is unknown whether they might develop SVD in the future. In addition, such databases include affected populations from diseases such as dementia, which might include patients with 
SVD due to misclassification. Determining whether variants segregate with disease in families is important but often family members are unavailable.

The use of targeted HTS panels presents several advantages. While WGS, whole-exome sequencing (WES), and HTS panels can all provide clinically acceptable coverage, the higher read depth of HTS panels allows for the detection of high-confidence CNVs. HTS panels also allow for savings in terms of cost and analysis time, compared to WGS and WES.

Our study has limitations. Our analyses did not include sequencing a cohort of MRI-phenotyped unaffected individuals. Previous studies have used age- and sex-matched controls unscreened for disease as comparison. ${ }^{40}$ However, the value of such controls is questionable. Much of SVD is subclinical prior to cerebrovascular events and ruling out subclinical disease in such controls is not possible.

This study was performed in patients of European ancestry. It would not be possible to extrapolate these results to populations of other ancestries as variant frequencies may vary significantly in different populations. Similar studies should be performed in other populations, particularly as population databases expand to better represent these ethnicities.

Our results demonstrate that monogenic mutations account for about $1.5 \%$ of SVD stroke. Furthermore, they demonstrate the utility of targeted HTS platforms in the diagnosis of monogenic forms of SVD. We showed they could detect CADASIL-causing variants, suggesting they could replace commonly used Sanger sequencing techniques, with the added benefit of screening for other genes, such as HTRAl, shown in this study to be the second most common cause of monogenic SVD.

\section{Acknowledgment}

The authors thank the contributors to the DNA Lacunar Stroke Study, listed at journals.plos.org/plosone/article? $\mathrm{id}=10.1371 /$ journal.pone. 0136352 . This study makes use of data generated by the NIHR BioResource-Rare Diseases. The authors thank the NIHR BioResource volunteers, the NIHR BioResource Centre, and its staff; the National Institute for Health Research; and NHS Blood and Transplant.

\section{Study funding}

Funding for the project was provided by the National Institute for Health Research (NIHR, grant RG65966). This work was supported by a BHF Programme Grant (RG/16/4/32218). Infrastructural support was provided by the Cambridge University Hospitals Trust Biomedical Research Centre. R.Y.Y.T. is supported by the Agency for Science, Technology and Research (Singapore). K.D. is a HSST trainee supported by NHS Health Education England. H.S.M. is supported by an NIHR Senior Investigator award.

\section{Disclosure}

The authors report no disclosures relevant to the manuscript. Go to Neurology.org/N for full disclosures.

\section{Publication history}

Received by Neurology January 10, 2019. Accepted in final form June 10, 2019.

Appendix Authors

\begin{tabular}{|c|c|c|c|}
\hline Name & Location & Role & Contribution \\
\hline $\begin{array}{l}\text { Rhea Y.Y. } \\
\text { Tan }\end{array}$ & $\begin{array}{l}\text { University } \\
\text { of } \\
\text { Cambridge }\end{array}$ & Author & $\begin{array}{l}\text { Study design, data acquisition, } \\
\text { data analysis, drafting of } \\
\text { manuscript }\end{array}$ \\
\hline $\begin{array}{l}\text { Matthew } \\
\text { Traylor }\end{array}$ & $\begin{array}{l}\text { University } \\
\text { of } \\
\text { Cambridge }\end{array}$ & Author & $\begin{array}{l}\text { Data analysis, drafting of } \\
\text { manuscript }\end{array}$ \\
\hline Karyn Megy & $\begin{array}{l}\text { University } \\
\text { of } \\
\text { Cambridge }\end{array}$ & Author & $\begin{array}{l}\text { Data acquisition, data analysis, } \\
\text { drafting of manuscript }\end{array}$ \\
\hline $\begin{array}{l}\text { Daniel } \\
\text { Duarte }\end{array}$ & $\begin{array}{l}\text { University } \\
\text { of } \\
\text { Cambridge }\end{array}$ & Author & Data acquisition \\
\hline $\begin{array}{l}\text { Sri V.V. } \\
\text { Deevi }\end{array}$ & $\begin{array}{l}\text { University } \\
\text { of } \\
\text { Cambridge }\end{array}$ & Author & Data acquisition, data analysis \\
\hline $\begin{array}{l}\text { Olga } \\
\text { Shamardina }\end{array}$ & $\begin{array}{l}\text { University } \\
\text { of } \\
\text { Cambridge }\end{array}$ & Author & Data acquisition, data analysis \\
\hline $\begin{array}{l}\text { Rutendo P. } \\
\text { Mapeta }\end{array}$ & $\begin{array}{l}\text { University } \\
\text { of } \\
\text { Cambridge }\end{array}$ & Author & Data acquisition \\
\hline $\begin{array}{l}\text { Willem H. } \\
\text { Ouwehand }\end{array}$ & $\begin{array}{l}\text { University } \\
\text { of } \\
\text { Cambridge }\end{array}$ & Author & $\begin{array}{l}\text { Design and conceptualization of } \\
\text { study }\end{array}$ \\
\hline Stefan Gräf & $\begin{array}{l}\text { University } \\
\text { of } \\
\text { Cambridge }\end{array}$ & Author & Data analysis \\
\hline $\begin{array}{l}\text { Kate } \\
\text { Downes }\end{array}$ & $\begin{array}{l}\text { University } \\
\text { of } \\
\text { Cambridge }\end{array}$ & Author & $\begin{array}{l}\text { Design and conceptualization of } \\
\text { study, data analysis, drafting of } \\
\text { manuscript }\end{array}$ \\
\hline $\begin{array}{l}\text { Hugh S. } \\
\text { Markus }\end{array}$ & $\begin{array}{l}\text { University } \\
\text { of } \\
\text { Cambridge }\end{array}$ & Author & $\begin{array}{l}\text { Design and conceptualization of } \\
\text { study, data analysis, drafting of } \\
\text { manuscript }\end{array}$ \\
\hline
\end{tabular}

\section{References}

1. Pantoni L. Cerebral small vessel disease: from pathogenesis and clinical characteristics to therapeutic challenges. Lancet Neurol 2010;9:689-701.

2. O'Brien JT, Thomas A. Vascular dementia. Lancet 2015;386:1698-1706.

3. Tan R, Traylor M, Rutten-Jacobs L, Markus H. New insights into mechanisms of small vessel disease stroke from genetics. Clin Sci 2017;131:515-531.

4. Chabriat H, Joutel A, Dichgans M, Tournier-Lasserve E, Bousser M-G. CADASIL. Lancet Neurol 2009;8:643-653.

5. Tan RY, Markus HS. Monogenic causes of stroke: now and the future. J Neurol 2015; 262:2601-2616.

6. Joutel A, Vahedi K, Corpechot C, et al. Strong clustering and stereotyped nature of Notch3 mutations in CADASIL patients. Lancet 1997;350:1511-1515.

7. MacArthur JAL, Morales J, Tully RE, et al. Locus Reference Genomic: reference sequences for the reporting of clinically relevant sequence variants. Nucleic Acids Res 2014;42:D873-D878.

8. Simeoni I, Stephens JC, Hu F, et al. A high-throughput sequencing test for diagnosing inherited bleeding, thrombotic, and platelet disorders. Blood 2016;127: 2791-2803. 
9. Kilarski LL, Rutten-Jacobs LCA, Bevan S, et al. Prevalence of CADASIL and Fabry disease in a cohort of MRI defined younger onset lacunar stroke. PLoS One 2015;10: e0136352.

10. Adams HP, Bendixen BH, Kappelle LJ, et al. Classification of subtype of acute ischemic stroke: definitions for use in a multicenter clinical trial: TOAST: Trial of Org 10172 in Acute Stroke Treatment. Stroke 1993;24:35-41.

11. Fazekas F, Kleinert R, Offenbacher H, et al. Pathologic correlates of incidental MRI white matter signal hyperintensities. Neurology 1993;43:1683-1689.

12. Lek M, Karczewski KJ, Minikel EV, et al. Analysis of protein-coding genetic variation in 60,706 humans. Nature 2016;536:285-291.

13. Fokkema IF, Taschner PE, Schaafsma GC, Celli J, Laros JF, den Dunnen JT. LOVD v.2.0: the next generation in gene variant databases. Hum Mutat 2011;32:557-563.

14. Kircher M, Witten DM, Jain P, O’Roak BJ, Cooper GM, Shendure J. A general framework for estimating the relative pathogenicity of human genetic variants. Nat Genet 2014;46:310-315.

15. Joutel A, Corpechot C, Ducros A, et al. Notch3 mutations in CADASIL, a hereditary adult-onset condition causing stroke and dementia. Nature 1996;383:707-710.

16. Richards S, Aziz N, Bale S, et al. Standards and guidelines for the interpretation of sequence variants: a joint consensus recommendation of the American College of Medical Genetics and Genomics and the Association for Molecular Pathology. Genet Med 2015; 17:405-424.

17. Simeoni I, Shamardina O, Deevi SVV, et al. GRID: Genomics of Rare Immune Disorders: a highly sensitive and specific diagnostic gene panel for patients with primary immunodeficiencies. Biorxiv Epub 2018 Oct 18:431544.

18. Raczy C, Petrovski R, Saunders CT, et al. Isaac: ultra-fast whole-genome secondary analysis on Illumina sequencing platforms. Bioinformatics 2013;29:2041-2043.

19. Hara K, Shiga A, Fukutake T, et al. Association of HTRA1 mutations and familial ischemic cerebral small-vessel disease. N Engl J Med 2009;360:1729-1739.

20. Tateoka T, Onda H, Hirota K, et al. Unusual case of cerebral small vessel disease with a heterozygous nonsense mutation in HTRA1. J Neurol Sci 2016;362:144-146.

21. Verdura E, Hervé D, Scharrer E, et al. Heterozygous HTRA1 mutations are associated with autosomal dominant cerebral small vessel disease. Brain 2015;138: 2347-2358.

22. Lanfranconi S, Markus HS. COL $4 A 1$ mutations as a monogenic cause of cerebral small vessel disease: a systematic review. Stroke 2010;41:e513-e518.

23. Plaisier E, Chen Z, Gekeler F, et al. Novel COL4A1 mutations associated with HANAC syndrome: a role for the triple helical CB3[IV] domain. Am J Med Genet A 2010;152A:2550-2555.

24. Weng YC, Sonni A, Labelle-Dumais C, et al. COL4A1 mutations in patients with sporadic late-onset intracerebral hemorrhage. Ann Neurol 2012;71:470-477.

25. French CR, Seshadri S, Destefano AL, et al. Mutation of FOXC1 and PITX2 induces cerebral small-vessel disease. J Clin Invest 2014;124:4877-4881.

26. Kumar M, Chambers C, Dhamija R. Axenfeld-Rieger syndrome and leukoencephalopathy caused by a mutation in FOXC1. Pediatr Neurol 2017;66:113-114.

27. DiFrancesco JC, Novara F, Zuffardi O, et al. TREX1 C-terminal frameshift mutations in the systemic variant of retinal vasculopathy with cerebral leukodystrophy. Neurol Sci 2015;36:323-330.

28. Crow YJ, Chase DS, Lowenstein Schmidt J, et al. Characterization of human disease phenotypes associated with mutations in TREX1, RNASEH2A, RNASEH2B, RNASEH2C, SAMHD1, ADAR, and IFIH1. Am J Med Genet A 2015;167A:296-312.

29. Rice G, Newman WG, Dean J, et al. Heterozygous mutations in TREX1 cause familial chilblain lupus and dominant Aicardi-Goutières syndrome. Am J Hum Genet 2007; 80:811-815.

30. McGlasson S, Rannikmäe K, Bevan S, et al. Rare variants of the $3^{\prime}-5^{\prime}$ DNA exonuclease TREX1 in early onset small vessel stroke. Wellcome Open Res 2017;2:106.
31. Firth HV, Richards SM, Bevan AP, et al. DECIPHER: database of chromosomal imbalance and phenotype in humans using ensemble resources. Am J Hum Genet 2009;84:524-533.

32. Jensson O, Palsdottir A, Thorsteinsson L, Arnason A. The saga of cystatin C gene mutation causing amyloid angiopathy and brain hemorrhage: clinical genetics in Iceland. Clin Genet 1989;36:368-377.

33. Di Donato I, Bianchi S, Gallus GN, et al. Heterozygous mutations of HTRA1 gene in patients with familial cerebral small vessel disease. CNS Neurosci Ther 2017;23:759-765.

34. Nozaki H, Sekine Y, Fukutake T, et al. Characteristic features and progression of abnormalities on MRI for CARASIL. Neurology 2015;85:459-463.

35. Rolfs A, Böttcher T, Zschiesche M, et al. Prevalence of Fabry disease in patients with cryptogenic stroke: a prospective study. Lancet 2005;366:1794-1796.

36. Crutchfield KE, Patronas NJ, Dambrosia JM, et al Quantitative analysis of cerebral vasculopathy in patients with Fabry disease. Neurology 1998;50:1746-1749.

37. Lanthier S, Saposnik G, Lebovic G, Pope K, Selchen D, Moore DF. Prevalence of Fabry disease and outcomes in young Canadian patients with cryptogenic ischemic cerebrovascular events. Stroke 2017;48:1766-1772.

38. Wozniak MA, Kittner SJ, Tuhrim S, et al. Frequency of unrecognized Fabry disease among young European-American and African-American men with first ischemic stroke.

39. Karczewski KJ, Francioli L. The Genome Aggregation Database (gnomAD) [online] Boston: MacArthur Lab; 2017.

40. Chong M, O'Donnell M, Thijs V, et al. Mendelian genes and risk of intracerebral hemorrhage and small-vessel ischemic stroke in sporadic cases. Stroke 2017;48:2263-2265.

41. Rutten JW, Dauwerse HG, Gravesteijn G, et al. Archetypal NOTCH3 mutations frequent in public exome: implications for CADASIL. Ann Clin Transl Neurol 2016; 3:844-853.

42. Markus HS, Martin RJ, Simpson MA, et al. Diagnostic strategies in CADASIL. Neurology 2002;59:1134-1138.

43. Peters N, Opherk C, Bergmann T, Castro M, Herzog J, Dichgans M. Spectrum of mutations in biopsy-proven CADASIL: implications for diagnostic strategies. Arch Neurol 2005;62:1091-1094.

44. Opherk C, Peters N, Herzog J, Luedtke R, Dichgans M. Long-term prognosis and causes of death in CADASIL: a retrospective study in 411 patients. Brain 2004;127:2533-2539.

45. Liao Y-C, Hsiao C-T, Fuh J-L, et al. Characterization of CADASIL among the Han Chinese in Taiwan: distinct genotypic and phenotypic profiles. PLoS One 2015;10: e0136501.

46. Ishida C, Sakajiri K, Yoshita M, Joutel A, Cave-Riant F, Yamada M. CADASIL with a novel mutation in exon 7 of NOTCH3 (C388Y). Intern Med 2006;45:981-985.

47. Kim Y, Choi EJ, Choi CG, et al. Characteristics of CADASIL in Korea: a novel cysteine-sparing Notch3 mutation. Neurology 2006;66:1511-1516.

48. Moreton FC, Razvi SSM, Davidson R, Muir KW. Changing clinical patterns and increasing prevalence in CADASIL. Acta Neurol Scand 2014;130:197-203.

49. Söder S, Pöschl E. The NC1 domain of human collagen IV is necessary to initiate triple helix formation. Biochem Biophys Res Commun 2004;325:276-280.

50. Eckman CB, Mehta ND, Crook R, et al. A new pathogenic mutation in the APP gene (I716V) increases the relative proportion of A beta 42(43). Hum Mol Genet 1997;6: 2087-2089.

51. Revesz T, Holton JL, Lashley T, et al. Genetics and molecular pathogenesis of sporadic and hereditary cerebral amyloid angiopathies. Acta Neuropathol 2009;118: $115-130$.

52. Jurkat-Rott $\mathrm{K}$, Freilinger T, Dreier JP, et al. Variability of familial hemiplegic migraine with novel A1A2 Na+/K+-ATPase variants. Neurology 2004;62:1857-1861.

53. Xu X, Yang X, Wu Q, et al. Amplicon resequencing identified parental mosaicism for approximately $10 \%$ of "de novo" SCN1A mutations in children with Dravet syndrome. Hum Mutat 2015;36:861-872. 


\section{Neurology}

\section{How common are single gene mutations as a cause for lacunar stroke?: A targeted gene panel study}

Rhea Y.Y. Tan, Matthew Traylor, Karyn Megy, et al.

Neurology 2019;93;e2007-e2020 Published Online before print November 12, 2019

DOI 10.1212/WNL.0000000000008544

This information is current as of November 12, 2019

\section{Updated Information \&} Services

References

Subspecialty Collections

Permissions \& Licensing

\section{Reprints}

including high resolution figures, can be found at: http://n.neurology.org/content/93/22/e2007.full

This article cites 50 articles, 11 of which you can access for free at: http://n.neurology.org/content/93/22/e2007.full\#ref-list-1

This article, along with others on similar topics, appears in the following collection(s):

\section{All Cerebrovascular disease/Stroke}

http://n.neurology.org/cgi/collection/all_cerebrovascular_disease_strok e

All Genetics

http://n.neurology.org/cgi/collection/all_genetics

CADASIL

http://n.neurology.org/cgi/collection/cadasil

Stroke in young adults

http://n.neurology.org/cgi/collection/stroke_in_young_adults

Information about reproducing this article in parts (figures,tables) or in its entirety can be found online at:

http://www.neurology.org/about/about_the_journal\#permissions

Information about ordering reprints can be found online:

http://n.neurology.org/subscribers/advertise

Neurology ${ }^{\circledR}$ is the official journal of the American Academy of Neurology. Published continuously since 1951, it is now a weekly with 48 issues per year. Copyright Copyright ( 2019 The Author(s). Published by Wolters Kluwer Health, Inc. on behalf of the American Academy of Neurology.. All rights reserved. Print ISSN: 0028-3878. Online ISSN: 1526-632X.

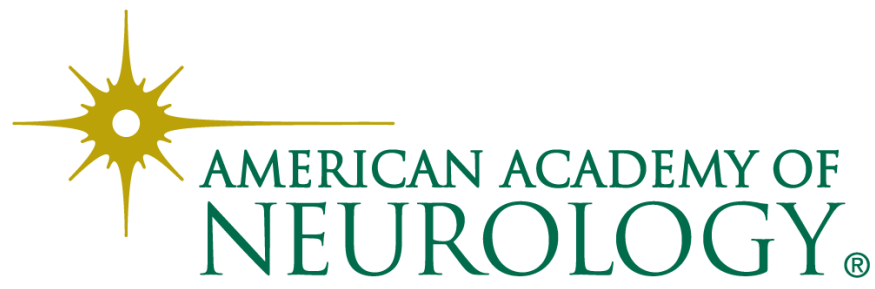

